\title{
Droplet Self-Propulsion on Superhydrophobic Microtracks
}

\section{Journal Article}

\section{Author(s):}

Stamatopoulos, Christos (D); Milionis, Athanasios; Ackerl, Norbert (D); Donati, Matteo; Leudet de la Vallée, Paul; Rudolf von Rohr, Philipp; Poulikakos, Dimos

Publication date:

2020-10-27

\section{Permanent link:}

https://doi.org/10.3929/ethz-b-000430943

\section{Rights / license:}

In Copyright - Non-Commercial Use Permitted

\section{Originally published in:}

ACS Nano 14(10), https://doi.org/10.1021/acsnano.0c03849 


\section{Droplet Self-Propulsion on Superhydrophobic Microtracks}

Christos Stamatopoulos," Athanasios Milionis, " Norbert Ackerl," Matteo Donati, Paul Leudet de la Vallée, Philipp Rudolf von Rohr,* and Dimos Poulikakos*

Cite This: ACS Nano 2020, 14, 12895-12904

ABSTRACT: Liquid transport (continuous or segmented) in microfluidic platforms typically requires pumping devices or external fields working collaboratively with special fluid properties to enable fluid motion. Natural liquid adhesion on surfaces deters motion and promotes the possibility of liquid or surface contamination. Despite progress, significant advancements are needed before devices for passive liquid propulsion, without the input of external energy and unwanted contamination, become a reality in applications. Here we present an unexplored and facile approach based on the Laplace pressure imbalance, manifesting itself through targeted track texturing, driving passively droplet motion, while maintaining the limited
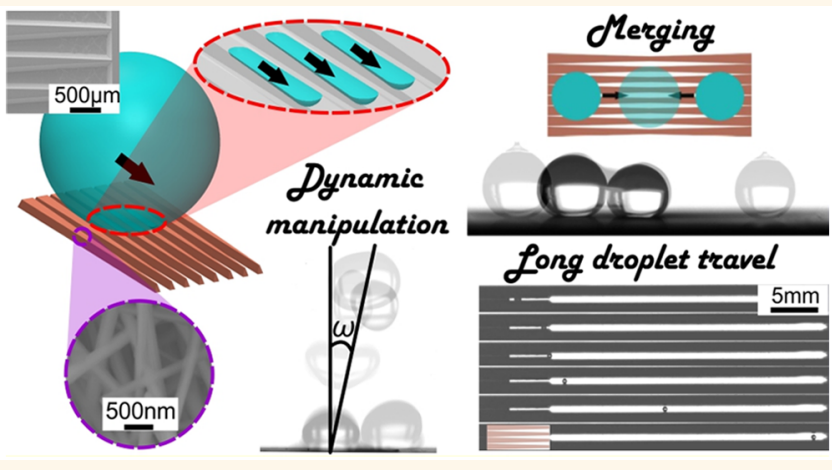
contact of the Cassie-Baxter state on superhydrophobic surfaces. The track topography resembles out-of-plane, backgammon-board, slowly converging microridges decorated with nanotexturing. This design naturally deforms asymmetrically the menisci formed at the bottom of a droplet contacting such tracks and causes a Laplace pressure imbalance that drives droplet motion. We investigate this effect over a range of opening track angles and develop a model to explain and quantify the underlying mechanism of droplet self-propulsion. We further implement the developed topography for applications relevant to microfluidic platform functionalities. We demonstrate control of the rebound angle of vertically impacting droplets, achieve horizontal self-transport to distances up to 65 times the droplet diameter, show significant uphill motion against gravity, and illustrate a self-driven droplet-merging process.

KEYWORDS: Laplace pressure imbalance, droplet self-propulsion, nano/microtexturing, superhydrophobicity, laser micromachining, droplet merging, drop impact

$\mathrm{D}$ irectional droplet mobility in so-called segmented flows is a desired functionality for a wide range of applications, such as microfluidics, ${ }^{1,2}$ on-chip devices, ${ }^{2}$ mixing for chemical processes, ${ }^{3,4}$ water collection from the atmosphere, ${ }^{5}$ and filtration. ${ }^{6}$ Traditionally, droplet generation and motion is achieved by using mechanical or electric power, such as in printing and pumping applications. ${ }^{7-9}$ Other active approaches have also been explored in the past to stimulate droplet motion, such as light, ${ }^{10,11}$ heat, ${ }^{12}$ magnetic, ${ }^{13}$ electric, ${ }^{14,15}$ or acoustic fields, ${ }^{16}$ often requiring tailored liquid properties to realize actuation.

Passive droplet propagation approaches are preferred over active ones, due to the absence of the need for energy input and their inherent facility. It has been shown in several manifestations in nature that Laplace pressure gradients can initiate and control liquid motion passively through surface structuring and chemistry, as exemplified by a spider silk, ${ }^{17}$ cactus spikes, ${ }^{18}$ a water strider's legs, ${ }^{19}$ and a pitcher plant. ${ }^{20}$ In all these cases, resulting wetting gradients play an important role in the demonstrated functionality.

Laplace pressure gradients through proper fabrication on surfaces have been shown to enhance the removal of condensate droplets on metallic textures ${ }^{21}$ and facilitate the removal of condensate. ${ }^{5,22}$ They can also be implemented to perform water-oil separation, ${ }^{6,23}$ affect the droplet dynamics at high temperatures, ${ }^{24}$ and, combined with surface lubrication, enhance droplet mobility. ${ }^{25}$ However, the existing surface designs that make use of Laplace pressure imbalance typically face the following challenges when dealing with transport of

Received: May 8, 2020

Accepted: July 29, 2020

Published: July 29, 2020 

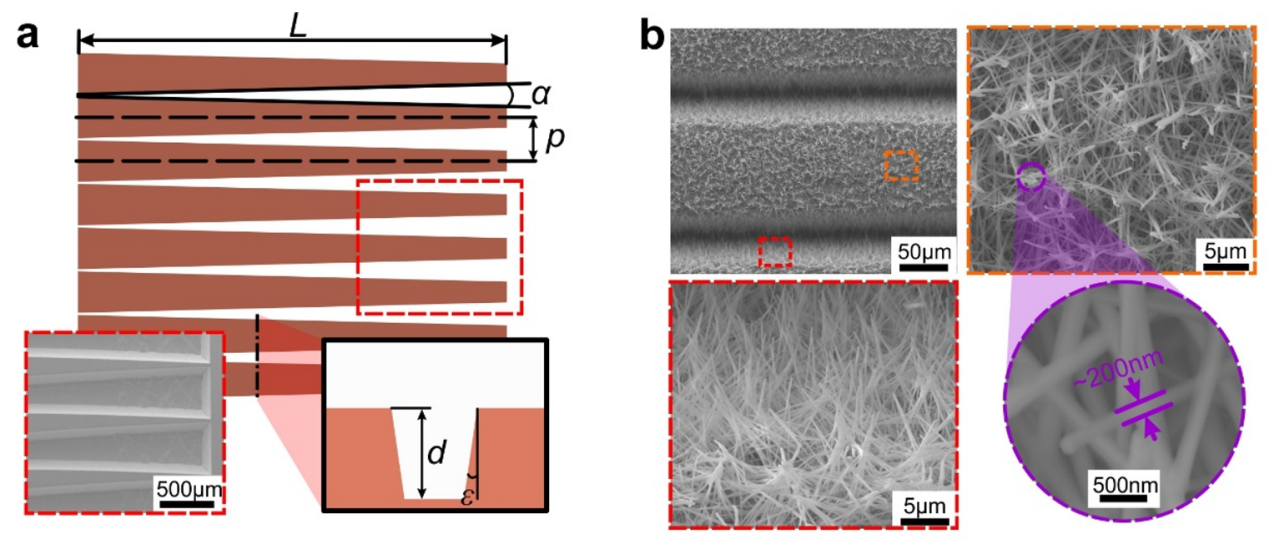

C
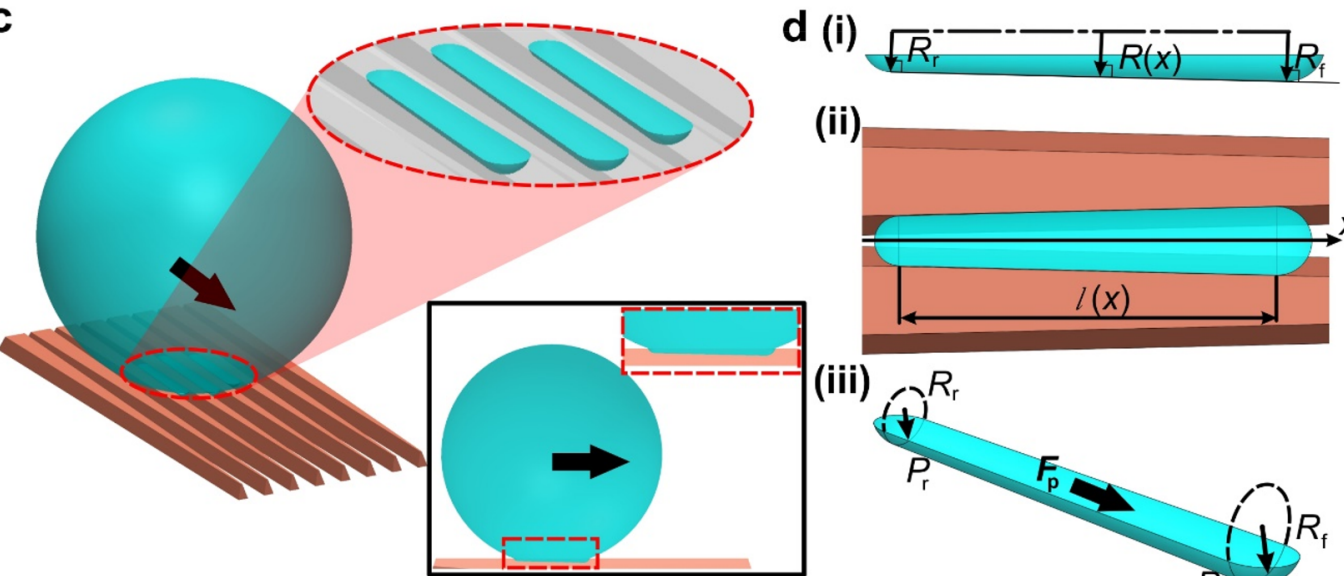

(ii)

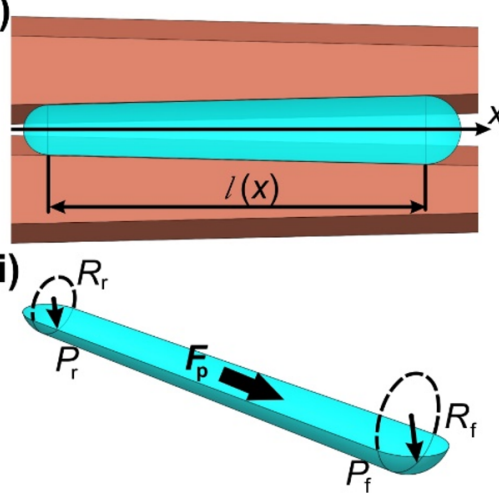

Figure 1. Surface design and droplet self-propulsion. (a) Schematic top view and geometrical parameters of the surface texture design: opening angle of the groove between two consecutive tracks $\alpha$, length of the entire structured surface $L$, and pitch $p$, i.e., the distance between the middle lines of two consecutive tracks. Left inset: SEM image of the texture for $\alpha=5^{\circ}$. Right inset: Schematic representation of a cut normal to the plane of the surface. The walls of the grooves with depth $d$ are at an angle $\varepsilon=13^{\circ}$. (b) SEM images showing the track and groove structures covered with a cluster of $\mathrm{Cu}(\mathrm{OH})_{2}$ nanoneedles. SEM images in dashed boxes show clustered nanoneedles on the top (orange dashed box) and the side walls (red dashed box) of the tracks. SEM in dashed purple shows a close-up of nanoneedles that exhibit a thickness of $200 \mathrm{~nm}$. (c) Schematic representation of the droplet when deposited on a surface. The bottom of the droplet deforms, and elongated menisci are formed. Inset: Vertical cut of the deformed droplet. (d) Schematic of the meniscus and associated geometrical considerations. (i) Side view of the meniscus. Its radius of curvature $R(\mathrm{x})$ changes linearly with $R_{\mathrm{r}}<R_{\mathrm{f}}$ (ii) Top view of the meniscus and its length $l(x)$. (iii) 3D illustration of the meniscus and radii of curvature at its rear and front parts. Differences in the radius of curvature result in a Laplace pressure gradient, which leads to a propulsion force $F_{\mathrm{p}}$ in the diverging direction of the groove.

small-sized droplets. First, the trajectory of the droplets cannot be controlled precisely, as in the case of coalesce-induced droplet jumping, ${ }^{21}$ where although no fluid loss occurs, the trajectories of the ejected droplets are random. On the other hand, when using channels for passive fluid transport, ${ }^{26-28}$ a certain amount of the fluid mass is lost or mixed during the process. These latter channels are based on fabricating a, usually, long and narrow hydrophilic region on an otherwise hydrophobic surface bounding this region. Then a liquid is deposited dropwise at one end of the hydrophilic region, which spreads within it through the combined action of surface hydrophilicity and a Laplace pressure gradient. Subsequent droplet deposition at one end of this region generates fluid transport again due to Laplace pressure imbalance, which forces a fluid mass to propagate along the already wetted area, thus expanding it. We note here that with this mechanism it is not the deposited mass that is propagated each time, but a given fraction of fluid mass that reaches the other end of the wet portion of the above-mentioned hydrophilic region. In addition, an unavoidable mixing occurs every time between the pre-existing liquid "pool" and the newly depositing drop at one end of it. Similar fabricated asymmetries on surface wetting properties of a surface can cause mixing and asymmetric bouncing. ${ }^{29}$ For the reasons stated above, such surfaces cannot achieve discretized transport if applied for example in the interior part of closed channels intended. Eventually, the most common method for discretized transport inside closed channels until now has been through two-phase flow systems, where the presence of two imiscible fluids is a prerequisite. ${ }^{30,31}$

Here we introduce and investigate a fully passive method, based on the nanoengineering of a superhydrophobic trackbased topography on copper surfaces. Our method is in stark contrast to the aforementioned approaches that offer relatively long, self-propagated, advancement of deposited liquid drops. Instead, through droplet self-propulsion, we demonstrate longdistance, directional, fluid transport without mass loss or mixing with predeposited liquid on surfaces and inside channels. The superhydrophobic copper surface is engineered using an easily scalable combination of laser micromachining (primary microstructures) and chemical etching (secondary nanostructures) followed by surface hydrophobization, initiating and sustaining droplet motion, driven solely by the 

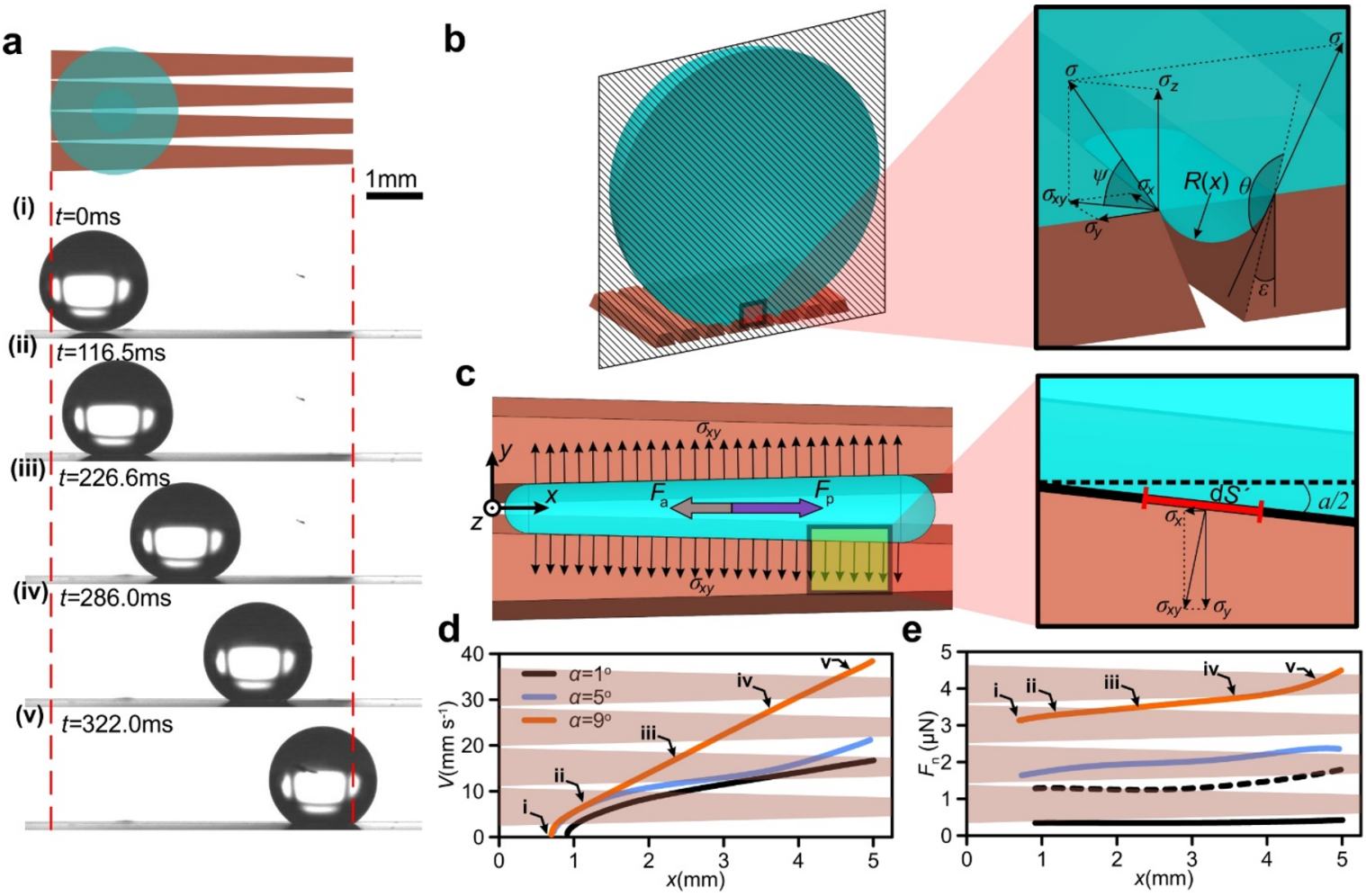

Figure 2. Droplet motion. (a) Time-lapse images (i) $-(v)$ of a $4 \mu \mathrm{L}$ droplet moving on a surface structure with $\alpha=9^{\circ}$. The droplet is released at a position that is $0.75 \mathrm{~mm}$ away from the beginning of the structuring (top schematic and $\mathrm{i}, t=0 \mathrm{~ms}$ ) and needs $322 \mathrm{~ms}$ to reach a position with the centroid being $0.25 \mathrm{~mm}$ away from the end of the structured area $(\mathrm{v}, t=322 \mathrm{~ms})$. The droplet travels a total distance of $4.25 \mathrm{~mm}$, i.e., 2.2 times the droplet diameter. (b) Cross section of a droplet (left) and associated 3D magnification of the meniscus (right). Resolution of adhesion force per unit length $\sigma$ into components $\sigma_{x}$ and $\sigma_{y}$ along the axes $x$ and $y$, respectively. (c) Top view of the meniscus (left). Two forces apply on the meniscus: the propulsion force $F_{\mathrm{p}}$ as a result of the Laplace pressure gradient along the meniscus and the adhesion force $F_{\mathrm{a}}$ as a result of the component $\sigma_{x}$ as can be seen at the magnification on the right. (d) Velocity $V$ versus $x$-direction for $\alpha=1^{\circ}, 5^{\circ}, 9^{\circ}$. Time instants $(i)-(v)$ correspond to the positions of the droplet shown in (a). (e) Net force $F_{n}=F_{p}-F_{a}$ exerted on one meniscus versus the $x$ direction for $\alpha=1^{\circ}, 5^{\circ}, 9^{\circ}$. Dashed line corresponds to the total force $F_{n, t o t a l}$ for $\alpha=1^{\circ}$ as a result of the formation of multiple menisci ( 5 in total). For the rest of the cases $\alpha=5^{\circ}, 9^{\circ}$ only one meniscus is formed. Time instants $(i)-(v)$ correspond to the positions of the droplet shown in (a).

Laplace-gradient force imposed, naturally by the texture design. We demonstrate that this platform can be used for efficient merging of fluids without mass loss and liquid transport at long distances much greater than the diameter of the transported droplet, also against gravity at significant elevations. It is important to mention that, according to our findings, it is enough to provide only a small portion of a superhydrophobic surface, where the droplet starts its journey, with the above mentioned microtracks. This generates a significant propulsion force, driving droplet self-transport also over the remainder of the same surface. These findings have the potential to generate possibilities for the realization of a broad range of passive microfluidic platforms.

\section{RESULTS AND DISCUSSION}

Working Principle. We texture a copper surface with a topography of hierarchical tracks upon which droplets are to be propelled. The shape of these tracks resembles closely the sides of severely acute, long, isosceles triangles, reminiscent of a backgammon board. Between every pair of such tracks a diverging microgroove is naturally formed (Figure 1a). It is the combined action of these microgrooves, when contacted by the liquid, that results in an overall capillary force imbalance able to induce droplet motion on the tracks, while sustaining the Cassie-Baxter superhydrophobic state.
The surface pattern is designed with the help of the following geometrical parameters defining the topography, namely, the opening angle $\alpha$, the pitch $p$, the depth $d$, and the length $L$ of the entire structured area (Figure 1a and right inset). In order to materialize the fabrication of these microridges, the associated design is introduced to a state-ofthe-art laser micromachining system (see Experimental Section). ${ }^{32,33}$ It should be noted that due to the shape of the laser beam used for the micromachining, an inherent nonzero sidewall angle $\varepsilon$ of the microridges is achieved, leading to a trapezoidal cross section (see Figure la left and right insets). This has a beneficial effect because it generates an upward capillary force aiding the superhydrophobic state of the droplet. To minimize the adhesion forces and facilitate selfpropulsion, we render the surface superhydrophobic by adding a second-level roughness to the tracks with the help of a cluster of $\mathrm{Cu}(\mathrm{OH})_{2}$ nanoneedles, with a thickness of $\sim 200 \mathrm{~nm}$ (see Figure $1 \mathrm{~b}) . .^{34-36}$ The clustered nanoneedles are then coated with a self-assembled monolayer (SAM), i.e., a perfluorodecanenthiol that reduces the surface energy of the substrate and renders it superhydrophobic (see Experimental Section). ${ }^{35,36}$ Typical advancing and receding contact angles of such a nanostructured surface are $\theta_{\text {rec }}=162.0^{\circ} \pm 1.6^{\circ}$ and $\theta_{\text {adv }}=$ $164.7^{\circ} \pm 1.0^{\circ}$, respectively, resulting in a contact angle hysteresis of $\Delta \theta=\theta_{\mathrm{adv}}-\theta_{\mathrm{rec}}=2.7 \pm 1.9^{\circ}$. 
A droplet placed on such a surface forms a series of elongated diverging menisci at its underside, as shown schematically in Figure 1c. The number of formed menisci depends on the size of the droplet and the design parameters of the tracks. Each such meniscus exhibits a linear dependence of its radius of curvature $R(x)$ with the $x$ direction (Figure $1 d(i))$ given by the equation

$$
R(x)=\left(R_{\mathrm{f}}-R_{\mathrm{r}}\right) / l(x) x+R_{\mathrm{r}}
$$

where $R_{\mathrm{r}}$ and $R_{\mathrm{f}}$ are the radii of curvature at the rear and front of the meniscus and $l(x)$ is its length (Figure $1 \mathrm{~d}(\mathrm{ii})$, see also Supporting Information, Discussion S1). This causes a Laplace pressure gradient along the meniscus, since $\Delta P(x)=P(x)-$ $P_{\text {air }}=\sigma / R(x)$, where $\Delta P(x)$ is the Laplace pressure difference, with $P(x)$ the pressure inside the meniscus at position $x, P_{\text {air }}$ the pressure of the surrounding air, and $\sigma$ the surface tension of water in air. The Laplace pressure gradient causes a propulsion force $F_{\mathrm{p}}$ in the diverging direction of the microgroove between two consecutive tracks with the internal pressures at the rear $P_{\mathrm{r}}$ and front $P_{\mathrm{f}}$ part of the meniscus, satisfying the condition $P_{\mathrm{r}}$ $>P_{\mathrm{f}}$ (Figure $\left.1 \mathrm{~d}(\mathrm{iii})\right) .{ }^{19,21,37-39}$ The propulsion force of the meniscus $F_{\mathrm{p}}$ is estimated by integrating this pressure gradient over the volume of the meniscus $\Omega^{\prime}$ (see Supporting Information, Discussion S1 section). ${ }^{37,40}$

Droplet Kinematics and Meniscus Dynamics. We studied the performance of three surfaces at three different opening angles, $\alpha=1^{\circ}, 5^{\circ}, 9^{\circ}$. For all the three surfaces, we kept the length the same, i.e., $L=5.25 \mathrm{~mm}$. We varied the pitch $p$ for each case, in order to regulate the number of formed menisci and accordingly affect the magnitude of the propulsion force $F_{\mathrm{p}}$. For $\alpha=1^{\circ}, 5^{\circ}, 9^{\circ}$ the pitches are $p=150,500,880$ $\mu \mathrm{m}$, respectively. We estimate that a droplet of volume of approximately $4 \mu \mathrm{L}$ forms five menisci for the case of $\alpha=1^{\circ}$ and one meniscus for the cases of $\alpha=5^{\circ}, 9^{\circ}$. The depth is chosen to be large enough, i.e., $d=240 \mu \mathrm{m}$ for the case of $\alpha=$ $1^{\circ}$ and $d=330 \mu \mathrm{m}$ for the cases of $\alpha=5^{\circ}, 9^{\circ}$, so that the formed menisci do not touch the bottom of the microgrooves. All the parameters used for each surface are summarized in Table S1.

Figure 2a shows time-lapse images of a droplet for the case of $\alpha=9^{\circ}$ (see also Video S1, where all the cases $\alpha=1^{\circ}, 5^{\circ}, 9^{\circ}$ are shown). A droplet of volume $4 \mu \mathrm{L}$ is released on all the surfaces with its centroid being $0.7 \mathrm{~mm}$ away from the beginning of the microgrooves. The droplet travels approximately a centroid to centroid distance of $4.25 \mathrm{~mm}$, i.e., 2.2 times the droplet diameter, in approximately $330 \mu \mathrm{s}$. The transport of the droplet occurs without any loss of mass. This further distinguishes the present work from existing concepts of liquid transport, such as that based on the different approach of adding liquid at the one end of a long, line-shaped, liquid pool to advance the liquid at the other end. $6,28,41,42$

The nanostructuring limits significantly the adhesion force $F_{a}$ which can be estimated with the help of Figure $2 \mathrm{~b}$. An adhesion force per unit length $\sigma$ is exerted at the triple phase (solid-air-liquid) line of the meniscus, which can be resolved into two components: the $\sigma_{z}$ is normal to the surface plane and the $\sigma_{x y}$ perpendicular to the triple phase line (Figure 2b, inset). The force per unit length $\sigma_{x y}$ can be further resolved into $\sigma_{x}$ and $\sigma_{y}$ parallel to the $x$ and $y$ axis, respectively (Figure $2 \mathrm{~b}$ and c). ${ }^{28,37}$ Thus, the adhesion force $F_{a}$ that resists the motion of the droplet along the $x$ axis can be estimated by integrating the $\sigma_{x}$ over two times the length of the ridge top edge (Figure 2c, see also Supporting Information, Discussion S1, for the derivation of the adhesion force).

With the combined effect of the nanostructuring and track microtexturing, the meniscus attains a shape that induces a favorable net force, $F_{\mathrm{n}}$, since $F_{\mathrm{n}}=F_{\mathrm{p}}-F_{\mathrm{a}}>0$ (Figure 2c), which is a necessary condition for the movement of the droplet. Indeed, as detailed in the Supporting Information, Discussion $\mathrm{S} 1$ section, the estimation of $F_{\mathrm{p}}$ and $F_{\mathrm{a}}$ suggests that $F_{\mathrm{p}}=l(x) \alpha \sigma \kappa_{\mathrm{p}}$ and $F_{\mathrm{a}}=l(x) \alpha \sigma \kappa_{\mathrm{a}}$ where $\kappa_{\mathrm{a}}$ and $\kappa_{\mathrm{p}}$ are constants. The constant $\kappa_{\mathrm{p}}$ is a function of $\psi$, and the constant $\kappa_{\mathrm{a}}$ is a function of $\psi$ and $\varphi$, where $\varphi$ is the fraction of the surface area in contact with the liquid (see Supporting Information, Discussion S1 section, about the estimation of the constants $\kappa_{\mathrm{a}}$ and $\left.\kappa_{\mathrm{p}}\right) .{ }^{43}$ Therefore, the net force $F_{\mathrm{n}}$ is given by the following equation:

$$
F_{\mathrm{n}}=l(x) \alpha \sigma\left(\kappa_{\mathrm{p}}-\kappa_{\mathrm{a}}\right)
$$

Equation 2 indicates that the necessary condition for the movement of the droplet is reduced to $\kappa_{\mathrm{p}}-\kappa_{\mathrm{a}}>0$, which can always be fulfilled for the specific micro/nanotexturing and resulting Cassie-Baxter state of the droplet meniscus (see also Supporting Information, Discussion S1).

Testing the three surfaces with three different opening angles and pitches confirms the validity of eq 2 and especially its strong dependence on opening angle $\alpha$. Based on the acquired time-lapse images and the distance traveled by the cross-sectional centroid of the droplet, we estimate the droplet velocity $V$ for the three tested surfaces, ${ }^{44}$ i.e., with $\alpha=1^{\circ}, 5^{\circ}, 9^{\circ}$ (Figure 2d). The velocity increases with $x$ and the droplet exhibits increasing velocities with opening angle, so that $V^{\alpha=9^{\circ}}$ $>V^{\alpha=5^{\circ}}>V^{\alpha=1^{\circ}}$. At the edge of the structures it attains values of $V=16.7,21.2$, and $38.4 \mathrm{~mm} \mathrm{~s}^{-1}$ for $\alpha=1^{\circ}, 5^{\circ}, 9^{\circ}$, respectively. The larger velocities with larger opening angle $\alpha$ are the result of the exerted force $F_{\mathrm{n}}$ that increases with $\alpha$ in the range investigated (see eq 2).

Moreover, we are able to quantify the force exerted on the meniscus of the droplet (Figure 2e). Equation 2 shows that the force scales with the length of the meniscus $l(x)$ as well (see Supporting Information, Discussion S2 for further details about the estimation of $l(x)) .^{40}$ It should be noted that for $\alpha=$ $1^{\circ}$, taking into consideration that five menisci are formed due to the droplet size and pitch $p$ used in this case, we show the calculated $F_{n}$ of the middle meniscus only (Figure $2 \mathrm{e}$, solid black line) as well as $F_{n, t o t a l}$ accounting for all five menisci (Figure 2e, dashed black line). Contrary to case $\alpha=1^{\circ}$, for the cases $\alpha=5^{\circ}, 9^{\circ}$ only one meniscus is formed. Further details on the estimation of $F_{n, t o t a l}$ are given in the Supporting Information, Discussion S3 section). Due to inherent limitations of the experimental setup, the number of menisici formed was not visually observed but is estimated using sideview video recordings and the conditions described in the Supporting Information, Discussion S3 section. The length of the meniscus for all three cases is estimated based on the diameter of the droplet's contact disc, as can be observed in the time lapse images. Here, the contact disc for all opening angles is intermittent, comprising interchanging solid-water interfaces and menisci/us (see Supporting Information, Discussions S2 and S3). Quantifying $F_{\mathrm{n}}$ for all cases shows that $F_{\mathrm{n}}^{\alpha=9^{\circ}}>$ $F_{\mathrm{n}}^{\alpha=5^{\circ}}>F_{\mathrm{n}, \text { total }}^{\alpha=1^{\circ}}>F_{\mathrm{n}}^{\alpha=1^{\circ}}$. At the end of the structured surface we obtain $F_{\mathrm{n}}^{\alpha=9^{\circ}}=4.5 \mu \mathrm{N}, F_{\mathrm{n}}^{\alpha=5^{\circ}}=2.4 \mu \mathrm{N}, F_{\mathrm{n}, \text { total }}^{\alpha=1^{\circ}}=1.8 \mu \mathrm{N}$, and 
a

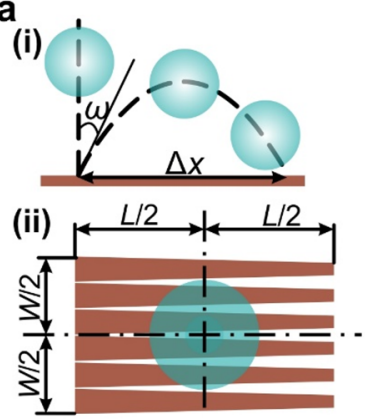

b

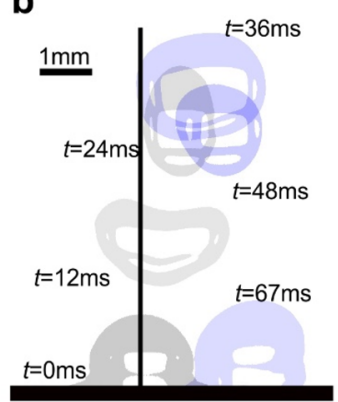

C

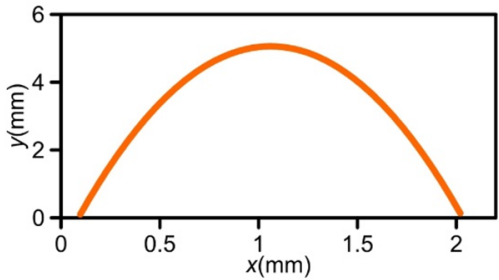

Figure 3. Drop impact on backgammon-like substrates. (a) Geometrical parameters of the drop impact experiments. (i) Parameters for the characterization of the droplet impact: rebound angle $\omega$ and displacement $\Delta x$. (ii) Position of the droplet impact. Droplet impact events. (b) Time-lapse images of a droplet impact and rebound for $\alpha=9^{\circ}$. Pseudocolored drop with blue corresponds to time instants $t \geq 36 \mathrm{~ms}$ where the drop reaches a maximum height, descends, and impacts again the surfaces. (c) Trajectory of the droplet between the first and second impact.

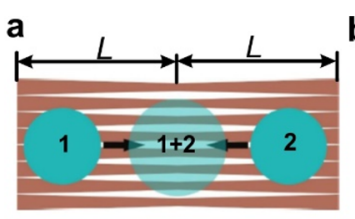

(iv)

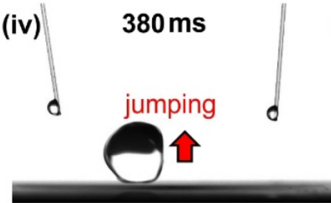

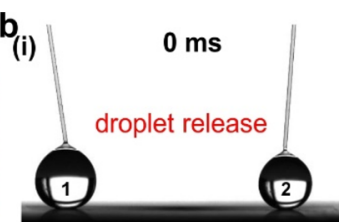
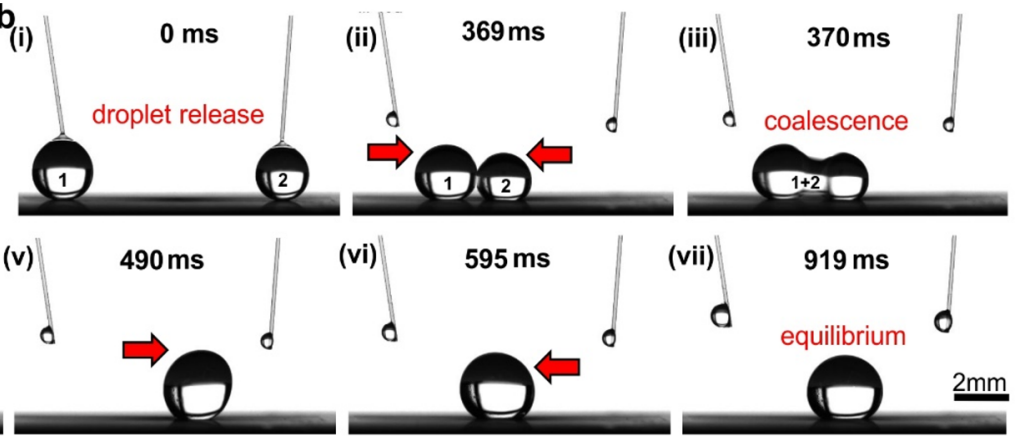

Figure 4. Droplet merging without liquid loss. (a) Top-view schematic of the mixing arrangement, which shows the position of the droplets upon release from capillaries, and the orientation of the microstructures to facilitate spontaneous, passive droplet merging. The black arrows depict the direction of the forces acting on the droplets. (b) High-speed frames from the droplet-merging process ((i) - (vii)). On the upper part of the frames, the time after the droplet release from the capillaries is indicated. Numbers (1 and 2$)$ are assigned to the droplets to facilitate distinction. The following steps are shown: (i) droplet release from the capillaries, (ii) approaching motion, (iii) coalescence, (iv) upward jumping, ( $\mathrm{v}$ and vi) back and forth oscillations until the excess kinetic energy is dissipated, and (vii) equilibrium.

$F_{\mathrm{n}}^{\alpha=1^{\circ}}=4.5 \mu \mathrm{N}$, which means that $F_{\mathrm{n}}^{\alpha=9^{\circ}}$ is $1.8,2.5$, and 11 times greater than $F_{\mathrm{n}}^{\alpha=5^{\circ}}, F_{\mathrm{n} \text {,total }}^{\alpha=1^{\circ}}$, and $F_{\mathrm{n}}^{\alpha=1^{\circ}}$, respectively (see Supporting Information, Discussions $\mathrm{S} 1-\mathrm{S} 3$, for further details on the calculation of $F_{\mathrm{n}}^{\alpha=5^{\circ}}, F_{\mathrm{n}}^{\alpha=9^{\circ}}, F_{\mathrm{n}, \text { total }}^{\alpha=1^{\circ}}$, and $F_{\mathrm{n}}^{\alpha=1^{\circ}}$ ).

It is worth noting here that the track opening angle design choice naturally depends on the application specifics and the targeted droplet advancement and velocity ranges. To this end, we performed an approximate analysis on the dependence of the resulting maximum $F_{\mathrm{n}}$ range on the opening angle. Our analysis supports that the opening angle $\alpha \approx 9^{\circ}$ is within the range around the maximum $F_{n}$ values (see Supporting Information, Discussion S2, for further information about the estimation analysis). In the following, we employ the opening angle $\alpha=9^{\circ}$ in the processes to be investigated.

Impact-Assisted Dynamic Manipulation of Droplets. Although manipulation of droplet rebound upon lateral impact on flat surfaces has been previously reported, ${ }^{29}$ here we achieve this through a different mechanism. Instead of using wettability gradients, we take advantage of the asymmetric meniscus deformation upon droplet impact, due to the specially designed microtrack arrays. We employ the surface with opening angle $\alpha=9^{\circ}$, which induces the largest horizontal force, and investigate its effect on imparting an off-vertical rebound trajectory after vertical drop impact. A drop is released from a height of $20 \mathrm{~mm}$. The contact time with the substrate is estimated to be $9 \mathrm{~ms}$. The drop rebound after impact is characterized by two parameters, namely, the rebound angle $\omega$, defined as the angle between the normal to the surface and the tangent to the rebound trajectory at the impact point, and the spatial displacement $\Delta x$, defined as the distance the drop centroid travels between the first two impact events, Figure $3 a(i)$. The impact point is at the axis of symmetry of the texture, as shown in Figure $3 a(i i)$. The expanded contact disc upon impact is contained within the structured area. The successive overlapping high-speed frames presented in Figure $3 \mathrm{~b}$ are taken every $12 \mathrm{~ms}$, starting from the first impact, until the moment that the droplet comes again into contact with the substrate after rebound. The Weber number based on the drop diameter $(1.87 \mathrm{~mm})$ is $W e \approx 11$ (see Video S2 for drop impact on a surface with opening angle $\alpha=9^{\circ}$ ).

Figure $3 \mathrm{~b}$ shows time-lapse images of a drop in the time period between the initial impact upon droplet release and the second impact after rebounding. We find that a significant deflection can be achieved, which is approximately equal to the diameter of the drop $(2 \mathrm{~mm})$, at a rebound angle $\omega=7.9^{\circ}$ (Figure 3c).

Droplet Merging for Liquid Mixing Applications. Next, we demonstrate droplet merging realized passively by taking advantage of the present surface structure as shown in Figure 4a. The droplet-merging setup included two capillaries that release two drops practically simultaneously. The surface structures with opening angles of $\alpha=9^{\circ}$ and length $L=5.25$ 


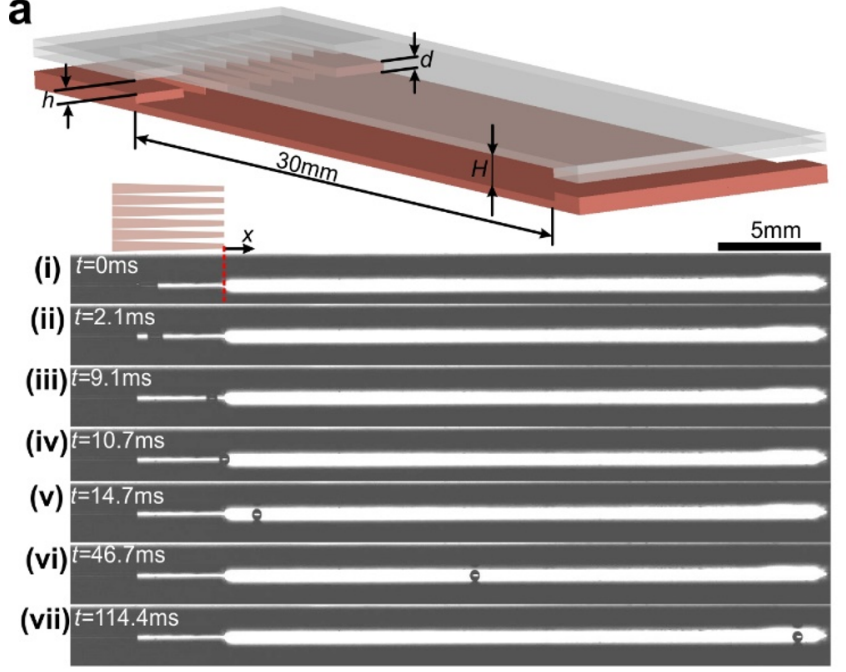

C

(i)

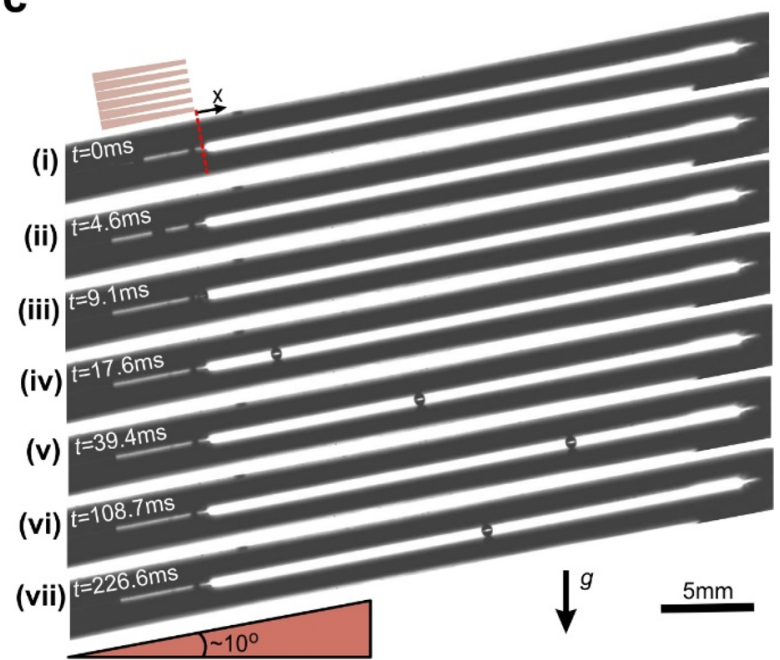

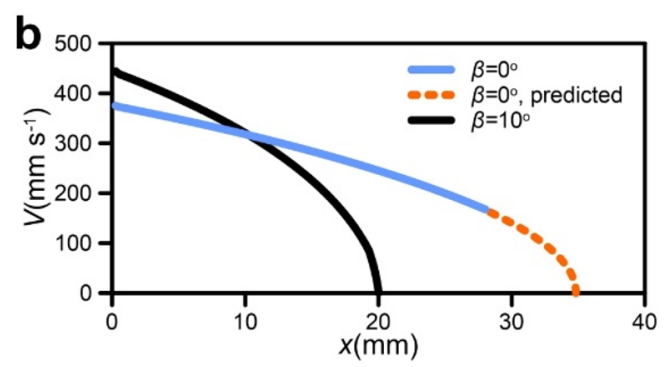

Figure 5. Droplet self-propulsion in microchannels. (a) Upper schematic: Microchannel configuration consisting of two segments: one segment microstructured and one planar superhydrophobic. Bottom panel: Time-lapse images (i) - (vii) showing a droplet of $\Omega=110 \mathrm{~nL}$ moving in the microchannel. The droplet gains momentum in the entry region (i)-(iv) and enters the planar region. The droplet reaches all the way to the end of the planar surfaces section, and its motion is interrupted due to limited channel length. (b) Droplet velocity $V$ (mm $\mathrm{s}^{-1}$ ) versus $x$-axis for horizontal $\beta=0^{\circ}$ (solid blue and dashed orange lines) and tilted $\beta=10^{\circ}$ (solid black line) microchannel. With the dashed orange line, we estimate the motion of the droplet in an infinitely long channel. (c) Time-lapse images (i) $-($ vii) showing a droplet of $\Omega=110 \mathrm{~nL}$ moving uphill in a microchannel tilted by $\beta=10^{\circ}$. The origin of the $x$-axis for both cases $\beta=0^{\circ}, 10^{\circ}$ coincides with the beginning of the planar surfaces section.

$\mathrm{mm}$ are placed in a mirroring arrangement, such that the Laplace pressure imbalance directs the droplets toward one another. Figure $4 \mathrm{a}$ shows the initial positions of the two droplets (1 and 2), approximately $0.75 \mathrm{~mm}$ from the boundaries of the structured area. Through the action of the Laplace pressure, the deposited droplets are directed to the central droplet-merging site ( $\operatorname{spot} 1+2)$.

The high-speed images in Figure $4 \mathrm{~b}(\mathrm{i})-($ vii) illustrate the various stages observed during the droplet-merging process (see also Video S3). After merging, we observe the phenomenon of coalescence-induced droplet jumping, which is known to occur only on superhydrophobic surfaces of very low adhesion. ${ }^{45}$ After approximately $920 \mathrm{~ms}$ from the droplet deposition, the droplet reaches an equilibrium when all the kinetic energy has been dissipated.

Self-Propelled Droplet Transport in Microchannels. An interesting aspect of the present concept of self-propelled droplets is in the context of a channel configuration (Figure 5a, upper schematic), as can be realized in on-chip applications. The closed channel configuration consists of two identical surfaces enclosing the droplet movement region. Along the length of the closed channel two regions are identified. The first region is the entry region of the channel, which is structured as discussed earlier, with $\alpha=9^{\circ}$, up to a length of $L$ $=5.25 \mathrm{~mm}$. The second region is the remainder of the channel with walls of length $30 \mathrm{~mm}$, significantly longer than the entry region. These walls are planar superhydrophobic surfaces without the self-propulsion texture, as shown in Figure 5. Their superhydrophibicity is imparted by growing clusters of nanoneedles covered with a perfluorodecanethiol layer, as in the entry self-propulsion regions. Other geometric parameters shown in Figure $5 \mathrm{a}$ are $h=140 \mu \mathrm{m}$ and $H=800 \mu \mathrm{m}$. With this configuration we double the force exerted on the droplet at the channel entrance, since both the top and the bottom of the droplet are in contact with it and deform the menisci found at these interfaces as mentioned above.

The droplet is released at the entrance of the microchannel with the help of a capillary tube at a position of approximately $1 \mathrm{~mm}$ after the beginning of the tracks. During the growth of the droplet two menisci (top and bottom) are formed. While growing, the adhesion force $F_{\mathrm{a} \text {,tip }}$ exerted from the tip of the capillary tube on the growing droplet and the adhesion force due to surface tension from the three-phase contact line of the droplet contact disc $F_{\mathrm{a}, \mathrm{cd}}$ dominate the total net force from the two surfaces, i.e., $F_{\mathrm{a}, \mathrm{tip}}+2 F_{\mathrm{a}, \mathrm{cd}}>2 F_{\mathrm{n}}$. Therefore, the droplet remains attached to the tip of the capillary tube. However, after some time, the length of the menisci $l$ increases, leading to an increase of $F_{n}$, as has been shown in the analysis in a previous section, until $F_{\mathrm{a}, \mathrm{tip}}+2 F_{\mathrm{a}, \mathrm{cd}}=2 F_{\mathrm{n}}$ (see Supporting Information, Discussion S4, regarding the associated force balance). Subsequently, the total net propulsion force in the axial direction surpasses $F_{\mathrm{a}, \mathrm{tip}}+2 F_{\mathrm{a}, \mathrm{cd}}$ and the droplet of volume $\Omega$ 
$=110 \mathrm{~nL}(\sim 600 \mu \mathrm{m}$ droplet diameter $)$ is released and starts its self-propelled travel (Figure $5 \mathrm{~b}$ ). The droplet needs only approximately $10.7 \mathrm{~ms}$ to travel along the length of the entrance region with microtracks (Figure 5a (i)-(iv)), after which it enters the planar section with a velocity of $V=375$ $\mathrm{mm} \mathrm{s}^{-1}$ and travels along the superhydrophobic planar surfaces a distance of $30 \mathrm{~mm}$ (Figure 5a (iv)-(vii); see also Video S4). It could clearly go further, but its motion is interrupted because the aforementioned section, and the channel comes to an end (see Figure $5 b$, blue solid line). To estimate the further distance that the droplet would have traveled if no physical obstacles had existed, we consider the droplet velocity $V$ estimated based on the centroid of the droplet similarly to what was described in the previous section. Our analysis shows that $V$ is a linear function of time $t$, denoting that the net force exerted on the droplet is constant. We also developed a model that estimates the relative magnitude of forces acting on the droplet, included in the Supporting Information, Discussion S4. The model suggests that viscous force is very small compared to the adhesion force originating from the bottom surfaces of the microchannel, which is in agreement with the existing literature. ${ }^{46-48}$ The contact disc of the droplet does not change, and therefore the adhesion force remains approximately constant. ${ }^{44}$ The estimated extra distance in a very long channel (no interference of the channel end) is shown in Figure $5 b$ (dashed orange line), measuring an impressive $39 \mathrm{~mm}$, which is 65 droplet diameters. To our knowledge this is by practically an order of magnitude the longest traveling distance reported taking into consideration that other existing concepts known to us are able to move a droplet by $8,{ }^{49} 4,{ }^{50}$ and $3^{51,52}$ droplet diameters.

We next test droplet motion against the action of gravity, which can add an important dimension in on-chip microfluidics, in terms of connecting passively device levels at different altitudes. ${ }^{53}$ The microchannel is tilted by $\beta=10^{\circ}$ (Figure 5c, see also Video S5), and to calculate the release size of the drop, the component of gravitational force along the $x$ axis is added to the adhesion force of the capillary tube tip, resisting the motion, i.e., $F_{\mathrm{a}, \mathrm{tip}}+2 F_{\mathrm{a}, \mathrm{cd}}+F_{\mathrm{g}, \mathrm{x}}$. A longer meniscus is of course required to overcome the total resisting force and start motion, i.e., $l^{\beta=10^{\circ}}>l^{\beta=0^{\circ}}$. This is confirmed by our experiments where a droplet of volume $\Omega=270 \mathrm{~nL}(\sim 800 \mu \mathrm{m}$ droplet diameter) is released (Figure $5 c(\mathrm{i})-($ iii)). We are also able to capture the mechanism of detachment and ejection described above, with a model the details of which are given in the Supporting Information, Discussion S4 section. Our model shows that in the limit where the droplet is attached on the needle by the natural capillary force during ejection and keeps growing until it detaches, the droplet will not detach for tilt angles $\beta>23^{\circ}$. In all experiments, however, if so desired, the force of the capillary tip can be easily eliminated by a quick withdrawal of the needle. In this case, we show that a drop can be ejected even when the microchannel is placed vertically, i.e., $\beta=90^{\circ}$. Subsequently, the droplet enters the section of the planar surfaces with a velocity of $V=440 \mathrm{~mm} \mathrm{~s}^{-1}$ (Figure $5 \mathrm{~b}$, solid black line; see also 5c(iii)) and is able to travel a distance of $20 \mathrm{~mm}$ along the section of planar surfaces (Figure 5c(iii)(vi)). Furthermore, it travels a distance of $4.25 \mathrm{~mm}$ along the entrance of the microstructured track section, Figure $5 \mathrm{c}$ (i)(iii)), covering a total distance of $24.25 \mathrm{~mm}$, which is an impressive 30 times the diameter of the droplet. Importantly, the droplet moves simultaneously against gravity, reaching an altitude increase of $4 \mathrm{~mm}$, which is 5 times the droplet diameter.

\section{CONCLUSION}

We introduced and investigated droplet self-propulsion on the basis of Laplace pressure gradients, realized by a surface texture composed out of out-of-plane, rationally designed superhydrophopic microtracks, which also maintained the droplet at the Cassie-Baxter state. We developed a model that explained the physical mechanism involved and were able to demonstrate self-propulsion distances, beyond the state of the art, both in the horizontal plane and in the direction opposite to gravity, as well as droplet merging and controlled off-vertical droplet rebound and hopping, all without liquid losses during transport. We believe that our proposed concept is a significant advancement for the further development of microfluidic platforms involving droplets, both in on-chip arrangements benefiting from passive segmented motion of liquids and in connecting such devices with passively moving droplets transitioning different elevations.

\section{EXPERIMENTAL SECTION}

Surface Fabrication. As-received copper samples of $1 \mathrm{~mm}$ thick (Cu CW004A Cu-ETP, Metall Service Menziken AG) are laser cut in a rectangular shape $20 \mathrm{~mm}$ wide and $50 \mathrm{~mm}$ long. Next, the trapezoidal microtracks are fabricated with laser micromachining in order to finally form the desired microtexture surface. The laser was set to $2 \mathrm{~W}$ average power at $1 \mathrm{MHz}$ repetition rate with $800 \mathrm{fs}$ pulses. A total number of 90 or 36 layers were ablated with an ablated layer thickness of $3.6 \mu \mathrm{m}$. A beam size of $2 \mu \mathrm{m}$ generates a surface roughness $\mathrm{Ra}<1 \mu \mathrm{m}$ and structural depth of 330 or $270 \mu \mathrm{m}$, respectively (see Supporting Information, Discussion S5 section, for further details about the laser micromachining).

Next, the microstructured copper sample was cleaned using ultrasonication by dipping it sequentially into baths of hydrochloric acid, acetone, isopropanol, and water. Then the sample was immersed in an aqueous solution of $77.5 \mathrm{mM}$ sodium hydroxide (Merck) and $3.1 \mathrm{mM}$ ammonium persulfate (Merck) for $25 \mathrm{~min}^{37}$ followed by immersion in an aqueous solution of $2.5 \mathrm{M}$ sodium hydroxide and 0.1 $\mathrm{M}$ ammonium persulfate for $7.5 \mathrm{~min} .^{35,36,54}$ After that, the sample is cleaned with water and dried using nitrogen. The outcome of this process is the formation of clustered copper hydroxide nanoneedles superimposed on the microtracks, resulting in a surface that has a hierarchical micro/nanotexture. Finally, we reduce the surface energy of the substrate by functionalization with perfluorodecanethiol. After the surface functionalization the substrate shows extreme water repellency.

Surface Characterization. For the optical examination of the surface morphology we used a scanning electron microscope (SEM, Quanta 200F FEI) as well as an optical microscope. We also employed energy-dispersive X-ray spectroscopy (EDX) with the system EDAX Octane Super embedded on the SEM, to perform chemical analysis of the backgammon-like surfaces. Associated microscope images and EDX graphs of the tested surfaces are given in the Supporting Information, Discussion S6 section. The planar samples, covered with hydrophobized clustered copper hydroxide nanoneedles, were characterized in terms of wettability. The advancing $\theta_{\text {adv }}$ and receding $\theta_{\text {rec }}$ contact angles were measured by depositing a $5 \mu \mathrm{L}$ water droplet at four different positions of the surface each time. For these measurements we employed the dynamic sessile drop technique with a commercial goniometer drop shape analyzer, DSA25 (Krüss).

Experimental Setup and Procedure. For the observation of the droplet self-propulsion, the merging of two drops, the drop impact experiments, and the movement of the drop inside a microchannel, we used a high-speed camera (FASTCAM Mini UX100, Photron) equipped with a macro lens (Nikon $200 \mathrm{~mm}$ f/4 AF-D). To ensure a 
uniform back-illumination and to optimize the contrast of the droplet shape, an LED backlight system with a light diffuser was used. Droplets were created with the help of a syringe pump (CETONI, BASE 120-neMESYS controller and neMESYS Low module V2) and were deposited on the targeted surfaces with a microcapillary. The surfaces were placed on a Pitch-and-Roll tilt platform (APR001/M, Thorlabs), which was mounted on an XYZ linear translational stage (MBT616D/M, Thorlabs). This enabled the manual control of the position of the samples with respect to the capillary and ensured that they were oriented horizontally. The vertical motion of the microcapillary was controlled electronically with a linear translational stage (M-404.4DG by PI) connected to a Mercury controller kit. A schematic of the experimental setup used is given in the Supporting Information, Figure S9.

For the droplet self-propulsion experiments the vertical capillary approached the tested surface to a distance of approximately $1.5 \mathrm{~mm}$. Then the syringe pump formed a droplet of approximately $1.9 \mathrm{~mm}$ diameter. The capillary moved upward with a speed of $0.1 \mathrm{~mm} / \mathrm{s}$, and the droplet due to gravity detached from the tip. With this approach, the vibrations induced on the droplet due to its abrupt detachment from the tip were reduced.

For the droplet-merging experiments we used a bifurcation in the main tube, which was connected to the syringe pump, at the end of which two branching tubes and two capillaries were connected, enabling the simultaneous deposition of two droplets on the tested surface.

The centroid of the droplets for the calculation of the droplet velocity was computed with the use of ImageJ software

\section{ASSOCIATED CONTENT}

\section{SI Supporting Information}

The Supporting Information is available free of charge at https://pubs.acs.org/doi/10.1021/acsnano.0c03849.

Video S1. Droplet self-propulsion on the surfaces with track opening angles $\alpha=1^{\circ}, 5^{\circ}, 9^{\circ}(\mathrm{MP} 4)$

Video S2. Drop impact on a surface with track opening angle $\alpha=9^{\circ}$ (MP4)

Video S3. Merging of two droplets. Track opening angle $\alpha=9^{\circ}$ (MP4)

Video S4. Ejection and movement of droplet inside a microchannel, track opening angle $\alpha=9^{\circ}$ (MP4)

Video S5. Ejection and movement of droplet inside a tilted microchannel (tilt anlge $\beta=10^{\circ}$ ), track opening angle $\alpha=9^{\circ}$ (MP4)

Derivation of net propulsion force $F_{n}$ calculation, estimation of meniscus length $l$ and parametric prediction of maximum net propulsion force $F_{n}$, estimation of total propulsion force $F_{n, t o t a l}$ for the case of multiple menisci formation, estimation of critical tilt angle $\beta_{\mathrm{cr}}$ laser micromachining process, surface characterization techniques (PDF)

\section{AUTHOR INFORMATION}

\section{Corresponding Authors}

Philipp Rudolf von Rohr - Transport Processes and Reactions Laboratory, ETH Zurich, 8092 Zurich, Switzerland;

Email: vonrohr@ipe.mavt.ethz.ch

Dimos Poulikakos - Laboratory of Thermodynamics in Emerging Technologies, ETH Zurich, 8092 Zurich, Switzerland; 이이.org/0000-0001-5733-6478; Email: dpoulikakos@ethz.ch

\section{Authors}

Christos Stamatopoulos - Transport Processes and Reactions Laboratory, ETH Zurich, 8092 Zurich, Switzerland; (1) orcid.org/0000-0002-8482-1150

Athanasios Milionis - Laboratory of Thermodynamics in Emerging Technologies, ETH Zurich, 8092 Zurich, Switzerland; 이이.org/0000-0002-0049-1255

Norbert Ackerl - Institute of Machine Tools and Manufacturing, Department of Mechanical and Process Engineering, ETH Zurich, 8092 Zurich, Switzerland; - orcid.org/0000-0001-5882-3243

Matteo Donati - Laboratory of Thermodynamics in Emerging Technologies, ETH Zurich, 8092 Zurich, Switzerland; (1) orcid.org/0000-0003-0197-0159

Paul Leudet de la Vallée - Laboratory of Thermodynamics in Emerging Technologies, ETH Zurich, 8092 Zurich,

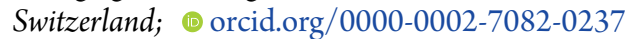

Complete contact information is available at:

https://pubs.acs.org/10.1021/acsnano.0c03849

\section{Author Contributions}

${ }$ C.S., A.M., and N.A. contributed equally.

\section{Author Contributions}

C.S. conceived the idea to perform this research. C.S., D.P., A.M., and P.Rv.R. designed and guided the research scientifically and technically throughout. C.S. and P.L.d.I.V. executed experiments. C.S., A.M., and A.N. analyzed data. C.S., M.D., and N.A. designed and fabricated the surfaces. C.S., A.M., N.A., D.P., and P.Rv.R. wrote the paper. The manuscript benefitted from contributions of all authors, who also have given approval to the final version of the manuscript.

\section{Notes}

The authors declare no competing financial interest.

\section{ACKNOWLEDGMENTS}

D.P., A.M., and M.D. have received funding from the European Union's Horizon 2020 research and innovation program under grant number 801229 (HARMoNIC). D.P., A.M., M.D., C.S., and P.R.v.R. acknowledge financial support from the Commission for Technology and Innovation (CTI) under the Swiss Competence Centers for Energy Research-Efficiency of Industrial Processes (SCCER-EIP) program (Grant No. KTI.2014.0148). N.A. received funding from the Swiss National Science Foundation (SNF) under grant number 169654 (FuSSI tools). We thank Dr. Karsten Kunze from the Scientific Center for Optical and Electron Microscopy, ETH Zurich, for his support in the acquisition of SEM and EDX data and Prof. Konrad Wegener, head of the Institute of Machine Tools and Manufacturing, ETH Zurich, for accommodating the fabrication of the tested surfaces in the facilities of the aforementioned institute. The authors thank Ms. Ines Weber and Mr. Marc Suter for their contribution to the preliminary study within the context of the associated project.

\section{REFERENCES}

(1) Caprioli, L.; Mele, E.; Angiì, F.E.; Girardo, S.; Athanassiou, A.; Camposeo, A.; Cingolani, R.; Pisignano, D. Photocontrolled Wettability Changes in Polymer Microchannels Doped with Photochromic Molecules. Appl. Phys. Lett. 2007, 91, 11-14. 
(2) Xing, S.; Harake, R. S.; Pan, T. Droplet-Driven Transports on Superhydrophobic-Patterned Surface Microfluidics. Lab Chip 2011, 11, 3642.

(3) Milionis, A.; Antonini, C.; Jung, S.; Nelson, A.; Schutzius, T. M.; Poulikakos, D. Contactless Transport and Mixing of Liquids on SelfSustained Sublimating Coatings. Langmuir 2017, 33, 1799-1809.

(4) Morrissette, J. M.; Mahapatra, P. S.; Ghosh, A.; Ganguly, R.; Megaridis, C. M. Rapid, Self-Driven Liquid Mixing on Open-Surface Microfluidic Platforms. Sci. Rep. 2017, 7, 1-13.

(5) Ghosh, A.; Beaini, S.; Zhang, B. J.; Ganguly, R.; Megaridis, C. M. Enhancing Dropwise Condensation through Bioinspired Wettability Patterning. Langmuir 2014, 30, 13103-13115.

(6) Mates, J. E.; Schutzius, T. M.; Qin, J.; Waldroup, D. E.; Megaridis, C. M. The Fluid Diode: Tunable Unidirectional Flow through Porous Substrates. ACS Appl. Mater. Interfaces 2014, 6, 12837-12843.

(7) Christensen, A. M.; Chang-Yen, D. A.; Gale, B. K. Characterization of Interconnects Used in PDMS Microfluidic Systems. J. Micromech. Microeng. 2005, 15, 928-934.

(8) Huang, S.; Li, C.; Lin, B.; Qin, J. Microvalve and Micropump Controlled Shuttle Flow Microfluidic Device for Rapid DNA Hybridization. Lab Chip 2010, 10, 2925-2931.

(9) Galliker, P.; Schneider, J.; Eghlidi, H.; Kress, S.; Sandoghdar, V.; Poulikakos, D. Direct Printing of Nanostructures by Electrostatic Autofocussing of Ink Nanodroplets. Nat. Commun. 2012, 3, 890.

(10) Monteleone, F. V.; Caputo, G.; Canale, C.; Cozzoli, P. D.; Cingolani, R.; Fragouli, D.; Athanassiou, A. Light-Controlled Directional Liquid Drop Movement on $\mathrm{TiO} 2$ Nanorods-Based Nanocomposite Photopatterns. Langmuir 2010, 26, 18557-18563.

(11) Makrygianni, M.; Millionis, A.; Kryou, C.; Trantakis, I.; Poulikakos, D.; Zergioti, I. On-Demand Laser Printing of PicoliterSized, Highly Viscous, Adhesive Fluids: Beyond Inkjet Limitations. Adv. Mater. Interfaces 2018, 5, 1-9.

(12) Mettu, S.; Chaudhury, M. K. Motion of Drops on a Surface Induced by Thermal Gradient and Vibration. Langmuir 2008, 24, 10833-10837.

(13) Timonen, J. V. I.; Latikka, M.; Leibler, L.; Ras, R. H. A.; Ikkala, O. Switchable Static and Dynamic Self-Assembly of Magnetic Droplets on Superhydrophobic Surfaces. Science 2013, 341, 253-257.

(14) Fan, S. K.; Huang, P. W.; Wang Te, T.; Peng, Y. H. Cross-Scale Electric Manipulations of Cells and Droplets by FrequencyModulated Dielectrophoresis and Electrowetting. Lab Chip 2008, 8, 1325-1331.

(15) Zhang, Y.; Wittstock, G. A Platform for Electric Field Aided and Wire-Guided Droplet Manipulation. Small 2017, 13, 1-6.

(16) Foresti, D.; Nabavi, M.; Klingauf, M.; Ferrari, A.; Poulikakos, D. Acoustophoretic Contactless Transport and Handling of Matter in Air. Proc. Natl. Acad. Sci. U. S. A. 2013, 110, 12549-12554.

(17) Zheng, Y.; Bai, H.; Huang, Z.; Tian, X.; Nie, F.-Q.; Zhao, Y.; Zhai, J.; Jiang, L. Directional Water Collection on Wetted Spider Silk. Nature 2010, 463, 640-643.

(18) Liu, C.; Xue, Y.; Chen, Y.; Zheng, Y. Effective Directional SelfGathering of Drops on Spine of Cactus with Splayed Capillary Arrays. Sci. Rep. 2015, 5, 1-8.

(19) Wang, Q.; Yao, X.; Liu, H.; Quéré, D.; Jiang, L. Self-Removal of Condensed Water on the Legs of Water Striders. Proc. Natl. Acad. Sci. U. S. A. 2015, 112, 47-52.

(20) Chen, H.; Zhang, P.; Zhang, L.; Liu, H.; Jiang, Y.; Zhang, D.; Han, Z.; Jiang, L. Continuous Directional Water Transport on the Peristome Surface of Nepenthes Alata. Nature 2016, 532, 85-89.

(21) Sharma, C. S.; Combe, J.; Giger, M.; Emmerich, T.; Poulikakos, D. Growth Rates and Spontaneous Navigation of Condensate Droplets through Randomly Structured Textures. ACS Nano 2017, $11,1673-1682$

(22) Tan, X.; Zhu, Y.; Shi, T.; Tang, Z.; Liao, G. Patterned Gradient Surface for Spontaneous Droplet Transportation and Water Collection: Simulation and Experiment. J. Micromech. Microeng. 2016, 26, 115009.
(23) Xu, W.; Lan, Z.; Peng, B.; Wen, R.; Chen, Y.; Ma, X. Directional Movement of Droplets in Grooves: Suspended or Immersed? Sci. Rep. 2016, 6, 1-11.

(24) Li, J.; Hou, Y.; Liu, Y.; Hao, C.; Li, M.; Chaudhury, M. K.; Yao, S.; Wang, Z. Directional Transport of High-Temperature Janus Droplets Mediated by Structural Topography. Nat. Phys. 2016, 12, 606-612.

(25) Zheng, Y.; Cheng, J.; Zhou, C.; Xing, H.; Wen, X.; Pi, P.; Xu, S. Droplet Motion on a Shape Gradient Surface. Langmuir 2017, 33, 4172.

(26) Deng, S.; Shang, W.; Feng, S.; Zhu, S.; Xing, Y.; Li, D.; Hou, Y.; Zheng, Y. Controlled Droplet Transport to Target on a High Adhesion Surface with Multi-Gradients. Sci. Rep. 2017, 7, 1-8.

(27) Khoo, H. S.; Tseng, F. G. Spontaneous High-Speed Transport of Subnanoliter Water Droplet on Gradient Nanotextured Surfaces. Appl. Phys. Lett. 2009, 95, 1-4.

(28) Ghosh, A.; Ganguly, R.; Schutzius, T. M.; Megaridis, C. M. Wettability Patterning for High-Rate, Pumpless Fluid Transport on Open, Non-Planar Microfluidic Platforms. Lab Chip 2014, 14, 15381550.

(29) Schutzius, T. M.; Graeber, G.; Elsharkawy, M.; Oreluk, J.; Megaridis, C. M. Morphing and Vectoring Impacting Droplets by Means of Wettability-Engineered Surfaces. Sci. Rep. 2015, 4, 7029.

(30) Gu, H.; Duits, M. H. G.; Mugele, F. Droplets Formation and Merging in Two-Phase Flow Microfluidics. Int. J. Mol. Sci. 2011, 12, 2572-2597.

(31) Asthana, A.; Zinovik, I.; Weinmueller, C.; Poulikakos, D. Significant Nusselt Number Increase in Microchannels with a Segmented Flow of Two Immiscible Liquids: An Experimental Study. Int. J. Heat Mass Transfer 2011, 54, 1456-1464.

(32) Ackerl, N.; Warhanek, M.; Gysel, J.; Wegener, K. UltrashortPulsed Laser Machining of Dental Ceramic Implants. J. Eur. Ceram. Soc. 2019, 39, 1635-1641.

(33) Ackerl, N.; Boerner, P.; Wegener, K. Toward Application of Hierarchical Structures by Ultrashort Pulsed Laser Ablation. J. Laser Appl. 2019, 31, 022501.

(34) Zhu, J.; Luo, Y.; Tian, J.; Li, J.; Gao, X. Clustered RibbedNanoneedle Structured Copper Surfaces with High-Efficiency Dropwise Condensation Heat Transfer Performance. ACS Appl. Mater. Interfaces 2015, 7, 10660-10665.

(35) Stamatopoulos, C.; Bleuler, P.; Pfeiffer, M.; Hedtke, S.; Von Rohr, P. R.; Franck, C. M. Influence of Surface Wettability on Discharges from Water Drops in Electric Fields. Langmuir 2019, 35 4876-4885.

(36) Stamatopoulos, C.; Sharma, C. S.; Suter, R.; Inäbnit, S.; Poulikakos, D.; Rudolf von Rohr, P. Surface Engineering for Heat Transfer Enhancement of Convective Dropwise Condensation. Proceedings of the 9th World Conference on Experimental Heat Transfer, Fluid Mechanics and Thermodynamics, Iguazu Falls, June 12-15, 2017.

(37) Sharma, C. S.; Stamatopoulos, C.; Suter, R.; Von Rohr, P. R.; Poulikakos, D. Rationally 3D-Textured Copper Surfaces for Laplace Pressure Imbalance-Induced Enhancement in Dropwise Condensation. ACS Appl. Mater. Interfaces 2018, 10, 29127-29135.

(38) Zheng, Y.; Bai, H.; Huang, Z.; Tian, X.; Nie, F.-Q.; Zhao, Y.; Zhai, J.; Jiang, L. Directional Water Collection on Wetted Spider Silk. Nature 2010, 463, 640-643.

(39) Zhang, C.; Zhang, B.; Ma, H.; Li, Z.; Xiao, X.; Zhang, Y.; Cui, X.; Yu, C.; Cao, M.; Jiang, L. Bioinspired Pressure-Tolerant Asymmetric Slippery Surface for Continuous Self-Transport of Gas Bubbles in Aqueous Environment. ACS Nano 2018, 12, 2048-2055.

(40) Renvoisé, P.; Bush, J. W. M.; Prakash, M.; Quéré, D. Drop Propulsion in Tapered Tubes. Europhys. Lett. 2009, 86, 64003.

(41) Li, J.; Zhou, X.; Li, J.; Che, L.; Yao, J.; McHale, G.; Chaudhury, M. K.; Wang, Z. Topological Liquid Diode. Sci. Adv. 2017, 3, 19-25.

(42) Li, J.; Feng, S.; Song, Y.; Wang, Z.; Zheng, H.; Xu, W. Designing Biomimetic Liquid Diode. Soft Matter 2019, 15, 19021915.

(43) Quéré, D. Wetting and Roughness. Annu. Rev. Mater. Res. 2008, $38,71-79$. 
(44) Stamatopoulos, C.; Schutzius, T. M.; Köppl, C. J.; Hayek El, N.; Maitra, T.; Hemrle, J.; Poulikakos, D. On the Shedding of Impaled Droplets: The Role of Transient Intervening Layers. Sci. Rep. 2016, 6, $1-9$.

(45) Boreyko, J. B.; Chen, C.-H. Self-Propelled Dropwise Condensate on Superhydrophobic Surfaces. Phys. Rev. Lett. 2009, 103, 184501.

(46) Wu, H.; Zhu, K.; Cao, B.; Zhang, Z.; Wu, B.; Liang, L.; Chai, G.; Liu, A. Smart Design of Wettability-Patterned Gradients on Substrate-Independent Coated Surfaces to Control Unidirectional Spreading of Droplets. Soft Matter 2017, 13, 2995-3002.

(47) Bliznyuk, O.; Jansen, H. P.; Kooij, E. S.; Zandvliet, H. J. W.; Poelsema, B. Smart Design of Stripe-Patterned Gradient Surfaces to Control Droplet Motion. Langmuir 2011, 27, 11238-11245.

(48) Suda, H.; Yamada, S. Force Measurements for the Movement of a Water Drop on a Surface with a Surface Tension Gradient. Langmuir 2003, 19, 529-531.

(49) Liu, C.; Sun, J.; Li, J.; Xiang, C.; Che, L.; Wang, Z.; Zhou, X. Long-Range Spontaneous Droplet Self-Propulsion on Wettability Gradient Surfaces. Sci. Rep. 2017, 7, 1-8.

(50) Lv, C.; Chen, C.; Chuang, Y. C.; Tseng, F. G.; Yin, Y.; Grey, F.; Zheng, Q. Substrate Curvature Gradient Drives Rapid Droplet Motion. Phys. Rev. Lett. 2014, 113, 1-5.

(51) Morgenthaler, S.; Lee, S.; Zürcher, S.; Spencer, N. D. A Simple, Reproducible Approach to the Preparation of Surface-Chemical Gradients. Langmuir 2003, 19, 10459.

(52) Neuhaus, S.; Padeste, C.; Spencer, N. D. Versatile Wettability Gradients Prepared by Chemical Modification of Polymer Brushes on Polymer Foils. Langmuir 2011, 27, 6855-6861.

(53) Shallan, A. I.; Priest, C. Microfluidic Process Intensification for Synthesis and Formulation in the Pharmaceutical Industry. Chem. Eng. Process. 2019, 142, 107559.

(54) Schlur, L.; Bonnot, K.; Spitzer, D. Synthesis of $\mathrm{Cu}(\mathrm{OH}) 2$ and $\mathrm{CuO}$ Nanotubes Arrays on a Silicon Wafer. RSC Adv. 2015, 5, 60616070 .

\section{NOTE ADDED AFTER ASAP PUBLICATION}

This paper published ASAP on August 6, 2020. In the Results and Discussion, value described as the typical receding contact angle was corrected to $164.7^{\circ}$. The revised paper was reposted on September 25, 2020. 